\title{
THE HAAGERUP PROPERTY FOR DRINFELD DOUBLES
}

\author{
SUTANU ROY
}

\begin{abstract}
We show that Drinfeld's double group construction for locally compact quantum groups preserves the Haagerup property. This shows that the Drinfeld doubles of the quantum groups, $\mathrm{C}_{0}\left(\mathbb{F}_{2}\right), \mathrm{SU}_{q}(2), \mathrm{SU}_{q}(1,1)$ ext, quantum $a x+b$, quantum $a z+b$, and $\mathrm{E}_{q}(2)$ have the Haagerup property.
\end{abstract}

\section{INTRODUCTION}

The Haagerup approximation property for groups is weaker than the notion of amenability. One of the equivalent formulations of the Haagerup property for groups is the existence of a mixing representation that weakly contains the trivial representation. Following this characterisation, Daws, Fima, Skalski and White introduced the Haagerup property for locally compact quantum groups in 7. Moreover, [7, Proposition 5.2] shows that the Haagerup property for a quantum group $\mathbb{G}$ follows from the coamenability of the dual quantum group $\widehat{\mathbb{G}}$. As an application, they show that quantum $a x+b$ in [20, quantum $a z+b$ in [21, and $\mathrm{E}_{q}(2)$ in [16], and their duals have the Haagerup property (see [7, Example 5.4]). All of these examples are amenable and coamenable. Also, like compact groups, all compact quantum groups have the Haagerup property. The quantum group $\mathrm{C}_{0}\left(\mathbb{F}_{2}\right)$ associated to the free group $\mathbb{F}_{2}$ is an example of a discrete non-amenable quantum group with the Haagerup property. The recent work of Caspers [6] shows that the extended $\mathrm{SU}_{q}(1,1)$ groups in [9], are examples of non-classical, non-discrete and non-amenable quantum groups that enjoy the Haagerup property. Moreover, their duals also have the Haagerup property.

The quantum double construction of Drinfeld or the Drinfeld double for Hopf algebras is one of the fundamental results of the pioneering work of Drinfeld [8. Roughly, the Drinfeld double of a finite dimensional Hopf algebra $H$ over a field $k$ is a Hopf algebra $\mathfrak{D}(H)$ such that the factors $H$ and $H^{*}:=\operatorname{Hom}_{k}(H, k)$ inside $\mathfrak{D}(H)$ do not commute. The quantum double construction for analytic quantum groups was developed in many different frameworks, along with the development of a general theory of compact and locally compact quantum groups. In 13, Podleś and Woronowicz introduced the double group construction, as the dual of the Drinfeld double, for compact quantum groups [18. In [2], Baaj and Vaes obtained Drinfeld double for regular (in the sense of Baaj and Skandalis [1) $\mathrm{C}^{*}$-algebraic locally compact quantum groups (see [10,11) as a special case of the dual of the generalised double crossed product construction.

In general, for $\mathrm{C}^{*}$-algebraic locally compact quantum groups (see [10,11]), this is generalised by Masuda, Nakagami and Woronowicz [11, Section 8], under the name quantum codouble. We shall follow this terminology. (What we call quantum codouble is called Drinfeld double in [12, Section 3]).

The main result of this article is the following theorem:

2010 Mathematics Subject Classification. Primary 81R50, Secondary 22D05, 46L65, 46L89.

Key words and phrases. Locally compact quantum groups, Haagerup property, Drinfeld double. 
Result 1.1. Drinfeld's double group construction preserves the Haagerup property. That is, the Drinfeld double of $\mathbb{G}$ has the Haagerup property whenever the quantum group $\mathbb{G}$ and its dual, $\widehat{\mathbb{G}}$ both have the Haagerup property.

After introducing basic definitions in Section 2, we briefly extract the $\mathrm{C}^{*}$-bialgebra structure from their construction in Section 3. In the last Section 4, we prove our main result 1.1. Then, we show that the Drinfeld doubles of the classical group $\mathbb{F}_{2}$, quantum $a x+b$, quantum $a z+b$, quantum $\mathrm{E}(2)$, quantum $\mathrm{SU}(2)$ and extended quantum $\mathrm{SU}(1,1)$ have the Haagerup property.

1.1. Basic notation. All Hilbert spaces and $\mathrm{C}^{*}$-algebras are assumed to be separable. For two norm-closed subsets $X$ and $Y$ of a $\mathrm{C}^{*}$-algebra, let

$$
X \cdot Y:=\{x y: x \in X, y \in Y\}^{\mathrm{CLS}},
$$

where CLS stands for the closed linear span.

For a $\mathrm{C}^{*}$-algebra $A$, let $\mathcal{M}(A)$ be its multiplier algebra and let $\mathcal{U}(A)$ be the group of unitary multipliers of $A$. Let $\mathfrak{C}^{*} \mathfrak{a l g}$ be the category of $\mathrm{C}^{*}$-algebras with nondegenerate ${ }^{*}$-homomorphisms $\varphi: A \rightarrow \mathcal{M}(B)$ as morphisms $A \rightarrow B$; let $\operatorname{Mor}(A, B)$ denote this set of morphisms.

For a Hilbert space $\mathcal{H}, \mathbb{K}(\mathcal{H})$ and $\mathbb{B}(\mathcal{H})$ denote the $\mathrm{C}^{*}$-algebras of compact and bounded operators acting on $\mathcal{H}$, respectively. A representation of a $\mathrm{C}^{*}$-algebra $A$ on a Hilbert space is an element of $\operatorname{Mor}(A, \mathbb{K}(\mathcal{H}))$. The group of unitary operators on a Hilbert space $\mathcal{H}$ is denoted by $\mathcal{U}(\mathcal{H})$.

We write $\Sigma$ for the tensor flip $\mathcal{H} \otimes \mathcal{K} \rightarrow \mathcal{K} \otimes \mathcal{H}, x \otimes y \mapsto y \otimes x$, for two Hilbert spaces $\mathcal{H}$ and $\mathcal{K}$. We write $\sigma$ for the tensor flip isomorphism $A \otimes B \rightarrow B \otimes A$ for two $\mathrm{C}^{*}$-algebras $A$ and $B$, where $\otimes$ denotes the minimal tensor product of $\mathrm{C}^{*}$-algebras.

\section{LOCALly COMPACT QUANTUM GROUPS}

For a general theory of $\mathrm{C}^{*}$-algebraic locally compact quantum groups see [10,11].

Definition 2.1 ([1, Définition 0.1]). A $\mathrm{C}^{*}$-bialgebra $\left(A, \Delta_{A}\right)$ is a $\mathrm{C}^{*}$-algebra $A$ and a comultiplication $\Delta_{A} \in \operatorname{Mor}(A, A \otimes A)$ that is coassociative: $\left(\operatorname{id}_{A} \otimes \Delta_{A}\right) \circ \Delta_{A}=$ $\left(\Delta_{A} \otimes \mathrm{id}_{A}\right) \circ \Delta_{A}$. Moreover, if $\Delta_{A}$ satisfies the cancellation property,

$$
\Delta_{A}(A) \cdot\left(1_{A} \otimes A\right)=\Delta_{A}(A) \cdot\left(A \otimes 1_{A}\right)=A \otimes A,
$$

$\left(A, \Delta_{A}\right)$ is a bisimplifiable $\mathrm{C}^{*}$-bialgebra.

Let $\varphi$ be a faithful (approximate) KMS weight (see [10, Section 1]) on $A$. The set of all positive $\varphi$-integrable elements is defined by $\mathcal{M}_{\varphi}^{+}:=\left\{a \in A^{+}: \varphi(a)<\infty\right\}$. Moreover, $\varphi$ is called

(1) left invariant if $\omega\left(\left(\operatorname{id}_{A} \otimes \varphi\right) \Delta_{A}(a)\right)=\omega(1) \varphi(a)$ for all $\omega \in A_{*}^{+}, a \in \mathcal{M}_{\varphi}^{+}$;

(2) right invariant if $\omega\left(\left(\varphi \otimes \operatorname{id}_{A}\right) \Delta_{A}(a)\right)=\omega(1) \varphi(a)$ for all $\omega \in A_{*}^{+}, a \in \mathcal{M}_{\varphi}^{+}$.

Definition 2.2 ([10, Definition 4.1]). A locally compact quantum group (quantum groups from now onwards) is a bisimplifiable $\mathrm{C}^{*}$-bialgebra $\mathbb{G}=\left(A, \Delta_{A}\right)$ with left and right invariant approximate $\mathrm{KMS}$ weights $\varphi$ and $\psi$, respectively.

By Theorem 7.14 and 7.15 in [10], the invariant weights $\varphi$ and $\psi$ are unique up to a positive scalar factor; hence they are called the left and right Haar weights for $\mathbb{G}$. Moreover, there is a unique (up to isomorphism) Pontrjagin dual $\widehat{\mathbb{G}}=\left(\hat{A}, \hat{\Delta}_{A}\right)$ of $\mathbb{G}$, which is again a quantum group.

Next we consider the GNS triple $\left(\mathrm{L}^{2}(\mathbb{G}), \pi, \Lambda\right)$ for $\psi$. There is a right multiplicative unitary $\mathbb{W} \in \mathcal{U}\left(\mathrm{L}^{2}(\mathbb{G}) \otimes \mathrm{L}^{2}(\mathbb{G})\right)$. Equivalently, $\mathbb{W}$ satisfies the pentagon equation:

$$
\mathbb{W}_{23} \mathbb{W}_{12}=\mathbb{W}_{12} \mathbb{W}_{13} \mathbb{W}_{23} \quad \text { in } \mathcal{U}\left(\mathrm{L}^{2}(\mathbb{G}) \otimes \mathrm{L}^{2}(\mathbb{G}) \otimes \mathrm{L}^{2}(\mathbb{G})\right)
$$


The right Haar weight version of the result [10, Proposition 6.10] ensures the manageability (see [15, Definition 2.1]) of $\mathbb{W}$. The theory of manageable multiplicative unitaries [19] gives:

(1) the dual multiplicative unitary $\widehat{\mathbb{W}}:=\Sigma \mathbb{W}^{*} \Sigma \in \mathcal{U}\left(\mathrm{L}^{2}(\mathbb{G}) \otimes \mathrm{L}^{2}(\mathbb{G})\right)$ is also manageable.

(2) the slices of $\mathbb{W}$ defined by

$$
\begin{aligned}
& A:=\left\{\left(\omega \otimes \mathrm{id}_{\mathrm{L}^{2}(\mathbb{G})}\right) \mathbb{W}: \omega \in \mathbb{B}\left(\mathrm{L}^{2}(\mathbb{G})\right)_{*}\right\}^{\mathrm{CLS}}, \\
& \hat{A}:=\left\{\left(\mathrm{id}_{\mathrm{L}^{2}(\mathbb{G})} \otimes \omega\right) \mathbb{W}: \omega \in \mathbb{B}\left(\mathrm{L}^{2}(\mathbb{G})\right)_{*}\right\}^{\mathrm{CLS}},
\end{aligned}
$$

are nondegenerate $\mathrm{C}^{*}$-subalgebras of $\mathbb{B}\left(\mathrm{L}^{2}(\mathbb{G})\right)$.

(3) $\mathbb{W} \in \mathcal{U}(\hat{A} \otimes A) \subseteq \mathcal{U}\left(\mathrm{L}^{2}(\mathbb{G}) \otimes \mathrm{L}^{2}(\mathbb{G})\right)$. We write $\mathrm{W}$ for $\mathbb{W}$ viewed as a unitary multiplier of $\hat{A} \otimes A$;

(4) the comultiplication maps $\Delta_{A}$ and $\hat{\Delta}_{A}$ are characterised by the following conditions:

$$
\begin{array}{ll}
\left(\mathrm{id}_{A} \otimes \Delta\right) \mathrm{W}=\mathrm{W}_{12} \mathrm{~W}_{13} & \text { in } \mathcal{U}(A \otimes \hat{A} \otimes \hat{A}) \\
\left(\hat{\Delta} \otimes \mathrm{id}_{A}\right) \mathrm{W}=\mathrm{W}_{23} \mathrm{~W}_{13} & \text { in } \mathcal{U}(\hat{A} \otimes \hat{A} \otimes A) .
\end{array}
$$

(5) there exist antiunitary involutive operators $J$ and $\hat{J}$ on $\mathrm{L}^{2}(\mathbb{G})$. They implement the unitary antipodes $\mathrm{R}$ and $\hat{R}$ on $\mathbb{G}$ and $\widehat{\mathbb{G}}$ as follows:

$$
\mathrm{R}(a):=\hat{J} a^{*} \hat{J} \quad \text { for } a \in A \quad \text { and } \quad \hat{\mathrm{R}}(\hat{a}):=J a^{*} J \quad \text { for } \hat{a} \in \hat{A} \text {. }
$$

The unitary $\mathrm{W} \in \mathcal{M}(\hat{A} \otimes A)$ is called the reduced bicharacter of $\mathbb{G}$.

Definition 2.6 ([4, Definition 3.1]). A quantum group $\mathbb{G}=\left(A, \Delta_{A}\right)$ is coamenable if it has a bounded counit. Equivalently, there is a unique ${ }^{*}$-homomorphism $e^{A} \in$ $\operatorname{Mor}(A, \mathbb{C})$ such that

$$
\left(e^{A} \otimes \mathrm{id}_{A}\right) \Delta_{A}=\mathrm{id}_{A} .
$$

\section{Duality Between quantum Codoubles and Drinfeld Doubles}

Let $\mathbb{G}=\left(A, \Delta_{A}\right)$ be a quantum group, let $\widehat{\mathbb{G}}=\left(\hat{A}, \hat{\Delta}_{A}\right)$ be its dual, and let $\mathrm{W} \in$ $\mathcal{U}(\hat{A} \otimes A)$ be the reduced bicharacter.

Define

$$
\widehat{\mathcal{D}}:=\hat{A} \otimes A \quad \text { and } \quad \hat{\Delta}_{\mathcal{D}}(\hat{a} \otimes a):=\left(\operatorname{id}_{\hat{A}} \otimes \sigma^{\mathrm{W}} \otimes \operatorname{id}_{A}\right)\left(\hat{\Delta}_{A} \otimes \Delta_{A}\right) .
$$

Here $\sigma^{\mathrm{W}} \in \operatorname{Mor}(\hat{A} \otimes A, A \otimes \hat{A})$ denotes the flip twisted by $\mathrm{W}$ defined by $\sigma^{\mathrm{W}}(\cdot):=$ $\sigma\left(\mathrm{W}^{*}(\cdot) \mathrm{W}\right)$. Define $\mathbb{U}:=\mathbb{W}(\hat{J} \otimes J) \mathbb{W}(\hat{J} \otimes J) \in \mathcal{U}\left(\mathrm{L}^{2}(\mathbb{G}) \otimes \mathrm{L}^{2}(\mathbb{G})\right)$. Theorem 8.7 in [11] shows that $\left(\widehat{\mathcal{D}}, \Delta_{\widehat{\mathcal{D}}}\right)$ is a quantum group and that

$$
\widehat{\mathbb{W}}^{\mathcal{D}}:=\mathbb{U}_{12} \widehat{\mathbb{W}}_{13} \mathbb{U}_{12}^{*} \mathbb{W}_{24} \in \mathcal{U}\left(\mathrm{L}^{2}(\mathbb{G}) \otimes \mathrm{L}^{2}(\mathbb{G}) \otimes \mathrm{L}^{2}(\mathbb{G}) \otimes \mathrm{L}^{2}(\mathbb{G})\right)
$$

is a manageable multiplicative unitary for it. The quantum codouble of $\mathbb{G}$, denoted by $\mathfrak{D}(\mathbb{G})^{\wedge}$, is the $\mathrm{C}^{*}$-bialgebra $\left(\widehat{\mathcal{D}}, \Delta_{\widehat{\mathcal{D}}}\right)$.

Definition 3.2. A pair of representations $\rho: A \rightarrow \mathbb{B}(\mathcal{H})$ and $\theta: \hat{A} \rightarrow \mathbb{B}(\mathcal{H})$ is called a $\mathbb{G}$-Drinfeld pair if it satisfies the $\mathbb{G}$-Drinfeld commutation relation:

$$
\mathrm{W}_{1 \rho} \mathrm{W}_{13} \mathrm{~W}_{\theta 3}=\mathrm{W}_{\theta 3} \mathrm{~W}_{13} \mathrm{~W}_{1 \rho} \quad \text { in } \mathcal{U}(\hat{A} \otimes \mathbb{K}(\mathcal{H}) \otimes A) \text {. }
$$

Here $\mathrm{W}_{1 \rho}:=\left(\left(\mathrm{id}_{\hat{A}} \otimes \rho\right) \mathrm{W}\right)_{12}$ and $\mathrm{W}_{\theta 3}:=\left(\left(\theta \otimes \mathrm{id}_{A}\right) \mathrm{W}\right)_{23}$.

Define $\rho: A \rightarrow \mathbb{B}\left(\mathrm{L}^{2}(\mathbb{G}) \otimes \mathrm{L}^{2}(\mathbb{G})\right)$ and $\theta: \hat{A} \rightarrow \mathbb{B}\left(\mathrm{L}^{2}(\mathbb{G}) \otimes \mathrm{L}^{2}(\mathbb{G})\right)$ by

$$
\rho(a):=\mathbb{U}\left(a \otimes 1_{\mathrm{L}^{2}(\mathbb{G})}\right) \mathbb{U}^{*} \quad \text { and } \quad \theta(\hat{a}):=1_{\mathrm{L}^{2}(\mathbb{G})} \otimes \hat{a} .
$$

Here we drop the GNS representations of $A$ and $\hat{A}$ on $\mathrm{L}^{2}(\mathbb{G})$. 
Proposition 3.4. The pair $(\rho, \theta)$ is a $\mathbb{G}$-Drinfeld pair. Define $\mathcal{D}:=\rho(A) \cdot \theta(\hat{A}) \subseteq$ $\mathbb{B}\left(\mathrm{L}^{2}(\mathbb{G}) \otimes \mathrm{L}^{2}(\mathbb{G})\right)$ and a map $\Delta_{\mathcal{D}}: \mathcal{D} \rightarrow \mathbb{B}\left(\mathrm{L}^{2}(\mathbb{G}) \otimes \mathrm{L}^{2}(\mathbb{G}) \otimes \mathrm{L}^{2}(\mathbb{G}) \otimes \mathrm{L}^{2}(\mathbb{G})\right)$ by

$$
\Delta_{\mathcal{D}}(\rho(a) \cdot \theta(\hat{a})):=(\rho \otimes \rho) \Delta_{A}(a)(\theta \otimes \theta) \hat{\Delta}_{A}(\hat{a}) \quad \text { for } a \in A, \hat{a} \in \hat{A} .
$$

Then $\left(\mathcal{D}, \Delta_{\mathcal{D}}\right)$ is the dual quantum group of the quantum codouble $\mathfrak{D}(\mathbb{G})^{\wedge}$.

Proof. The $\mathbb{G}$-Drinfeld commutation relation for the pair $(\rho, \theta)$ is equivalent to the following relation

$$
\mathbb{U}_{23} \mathbb{W}_{12} \mathbb{U}_{23}^{*} \mathbb{W}_{14} \mathbb{W}_{34}=\mathbb{W}_{34} \mathbb{W}_{14} \mathbb{U}_{23} \mathbb{W}_{12} \mathbb{U}_{23}^{*}
$$

then is one the intermediate steps in the proof of Proposition 8.6 in [11.

The multiplicative unitary in (3.1) is $\widehat{\mathbb{W}}^{\mathcal{D}}=\widehat{\mathbb{W}}_{\rho 2} \mathbb{W}_{\theta 3}$. The manageability of $\mathbb{W}$ and $\widehat{\mathbb{W}}$ imply:

$$
\left\{\left(\operatorname{id}_{\mathrm{L}^{2}(\mathbb{G}) \otimes \mathrm{L}^{2}(\mathbb{G})} \otimes \omega \otimes \omega^{\prime}\right) \widehat{\mathbb{W}}_{\rho 2} \mathbb{W}_{\theta 3}: \omega, \omega^{\prime} \in \mathbb{B}\left(\mathrm{L}^{2}(\mathbb{G})\right)_{*}\right\}^{\mathrm{CLS}}=\theta(\hat{A}) \cdot \rho(A)=\mathcal{D} .
$$

Since $\widehat{\mathbb{W}}^{\mathcal{D}}$ is also manageable, $\mathcal{D}$ is a nondegenerate $\mathrm{C}^{*}$-subalgebra of $\mathbb{B}\left(\mathrm{L}^{2}(\mathbb{G}) \otimes\right.$ $\left.\mathrm{L}^{2}(\mathbb{G})\right)$.

Furthermore, we get $\widehat{\mathbb{W}}_{\rho 2} \mathbb{W}_{\theta 3} \in \mathcal{U}(\mathcal{D} \otimes \widehat{\mathcal{D}})$.

The definition of $\Delta_{\mathcal{D}}$ gives $\Delta_{\mathcal{D}} \in \operatorname{Mor}(\mathcal{D}, \mathcal{D} \otimes \mathcal{D})$. Hence it is sufficient to check that $\Delta_{\mathcal{D}}$ satisfies (2.5) for $\mathrm{W}=\widehat{\mathbb{W}}_{\rho 2} \mathbb{W}_{\theta 3}$. We compute

$$
\begin{aligned}
\left(\Delta_{\mathcal{D}} \otimes \operatorname{id}_{\hat{A} \otimes A}\right) \widehat{\mathbb{W}}_{\rho 2} \mathbb{W}_{\theta 3} & =\left(\left((\rho \otimes \rho) \hat{\Delta} \otimes \operatorname{id}_{\hat{A}}\right) \widehat{\mathbb{W}}\right)_{123}\left(\left((\theta \otimes \theta) \Delta \otimes \operatorname{id}_{A}\right) \mathbb{W}\right)_{124} \\
& =\widehat{\mathbb{W}}_{\rho_{1} 3} \widehat{\mathbb{W}}_{\rho_{2} 3} \mathbb{W}_{\theta_{1} 4} \mathbb{W}_{\theta_{2} 4}=\widehat{\mathbb{W}}_{\rho_{1} 3} \mathbb{W}_{\theta_{1} 4} \widehat{\mathbb{W}}_{\rho_{2} 3} \mathbb{W}_{\theta_{2} 4} .
\end{aligned}
$$

The first equality follows from (3.5); the second equality uses (2.4) and (2.5), and $\rho_{i}$ or $\theta_{i}$ means $\rho$ or $\theta$ acting on the $i$-th leg for $i=1,2$; and the third equality uses the trivial commutation between $\widehat{\mathbb{W}}_{\rho_{2} 3}$ and $\mathbb{W}_{\theta_{1} 4}$.

Definition 3.6. The quantum group $\mathfrak{D}(\mathbb{G}):=\left(\mathcal{D}, \Delta_{\mathcal{D}}\right)$ is the Drinfeld double of $\mathbb{G}$.

Example 3.7. Let $A=\mathrm{C}_{0}(G)$ and $\hat{A}=\mathrm{C}_{\mathrm{r}}^{*}(G)$ for a locally compact group $G$. The underlying $\mathrm{C}^{*}$-algebra of the Drinfeld double of $G$ is $\left.\mathrm{C}(G)\right) \rtimes_{\text {conj }} G$.

\section{The HaAgerup property for the Drinfeld double}

A (unitary) representation of $\mathbb{G}=\left(A, \Delta_{A}\right)$ on a Hilbert space $\mathcal{H}$ is a unitary $\mathrm{V} \in \mathcal{U}(\mathbb{K}(\mathcal{H}) \otimes A)$ satisfying the following condition:

$$
\left(\mathrm{id}_{\mathcal{H}} \otimes \Delta_{A}\right) \mathrm{V}=\mathrm{V}_{12} \mathrm{~V}_{13} \quad \text { in } \mathcal{U}(\mathbb{K}(\mathcal{H}) \otimes A \otimes A) .
$$

Definition 4.2 ([7, Definition 5.1]). A locally compact quantum group $\mathbb{G}=$ $\left(A, \Delta_{A}\right)$ has the Haagerup property if there is a representation $\mathrm{V} \in \mathcal{U}(\mathbb{K}(\mathcal{H}) \otimes A)$ with the following properties:

(1) there is a net $\left\{x_{i}\right\}$ of almost invariant unit vectors in $\mathcal{H}$ :

$$
\left\|\mathrm{V}\left(x_{i} \otimes \eta\right)-x_{i} \otimes \eta\right\| \rightarrow 0 \quad \text { for all } \eta \in \mathrm{L}^{2}(\mathbb{G}) .
$$

(2) $\mathrm{V}$ is a mixing representation: $\left(\omega_{x, y} \otimes \operatorname{id}_{A}\right) \mathrm{U} \in A$ for all $x, y \in \mathcal{H}$, where $\omega_{x, y}(T):=\langle T x, y\rangle$ is the vector functional.

Next we prove our main result:

Theorem 4.4. Let $\mathbb{G}$ and $\widehat{\mathbb{G}}$ have the Haagerup property. Then the Drinfeld double of $\mathbb{G}$ also has the Haagerup property. 
Proof. Let $\mathrm{X} \in \mathcal{U}\left(\mathbb{K}\left(\mathcal{H}_{1}\right) \otimes A\right)$ and $\mathrm{Y} \in \mathcal{U}\left(\mathbb{K}\left(\mathcal{H}_{2}\right) \otimes \hat{A}\right)$ be representations of $\mathbb{G}$ and $\widehat{\mathbb{G}}$, respectively.

Define $\mathrm{V}:=\mathrm{X}_{1 \rho} \mathrm{Y}_{2 \theta} \in \mathcal{U}\left(\mathbb{K}\left(\mathcal{H}_{1} \otimes \mathcal{H}_{2}\right) \otimes \mathcal{D}_{\mathrm{V}}\right)$. Equations (3.5) and (4.1) for $\mathrm{X}$ and $\mathrm{Y}$ give:

$$
\left(\mathrm{id}_{\mathcal{H}_{1} \otimes \mathcal{H}_{2}} \otimes \Delta_{\mathcal{D}_{\mathrm{V}}}\right) \mathrm{X}_{1 \rho} \mathrm{Y}_{2 \theta}=\mathrm{X}_{1 \rho_{3}} \mathrm{X}_{1 \rho_{4}} \mathrm{Y}_{2 \theta_{3}} \mathrm{Y}_{2 \theta_{4}}=\mathrm{X}_{1 \rho_{3}} \mathrm{Y}_{2 \theta_{3}} \mathrm{X}_{1 \rho_{4}} \mathrm{Y}_{2 \theta_{4}}
$$

Hence $\mathrm{V}$ is a representation of the Drinfeld double $\mathfrak{D}(\mathbb{G})$ on $\mathcal{H}_{1} \otimes \mathcal{H}_{2}$.

Let $\mathrm{X}$ and $\mathrm{Y}$ satisfy the mixing condition (2) in Definition 4.2, Then we get the mixing condition for $\mathrm{V}$ :

$$
\left.\left(\omega_{x_{1} \otimes y_{1}, x_{2} \otimes y_{2}} \otimes \operatorname{id}_{\mathcal{D}_{\mathrm{V}}}\right)\left(\mathrm{X}_{1 \rho} \mathrm{Y}_{2 \theta}\right)=\rho\left(\left(\omega_{x_{1}, y_{1}} \otimes \operatorname{id}_{A}\right) \mathrm{X}\right) \theta\left(\omega_{y_{1}, y_{2}} \otimes \operatorname{id}_{\hat{A}}\right) \mathrm{Y}\right) \in \mathcal{D}_{\mathrm{V}}
$$

for all $x_{1}, x_{2} \in \mathcal{H}_{1}, y_{1}, y_{2} \in \mathcal{H}_{2}$.

Let $\mathbb{X} \in \mathcal{L}\left(\mathcal{H}_{1} \otimes A\right)$ be the image of $\mathrm{X} \in \mathcal{U}\left(\mathbb{K}\left(\mathcal{H}_{1}\right) \otimes A\right)$ under the canonical isomorphism between $\mathcal{M}\left(\mathbb{K}\left(\mathcal{H}_{1}\right) \otimes A\right)$ and $\mathcal{L}\left(\mathcal{H}_{1} \otimes A\right)$ (the space of adjointable operators on the Hilbert $A$-module $\mathcal{H}_{1} \otimes A$ ). By [7, Proposition 2.7], condition (4.3) becomes equivalent to the following condition:

$$
\left\|\mathbb{X}\left(x_{i} \otimes a\right)-x_{i} \otimes a\right\| \rightarrow 0 \quad \text { for all } a \in A .
$$

Let $\left\{x_{i}\right\}$ be the net of almost invariant unit vectors in $\mathcal{H}_{1}$ for X. Let $d \in \mathcal{D}_{\mathrm{V}}$ and let $\left\{e_{\lambda}^{A}\right\}$ be a bounded approximate identity in $A$. The construction of $\mathcal{D}$ gives $\rho \in \operatorname{Mor}(A, \mathcal{D})$. Given any $\epsilon>0$ there exists $\lambda^{\prime}$ such that $\left\|\rho\left(e_{\lambda^{\prime}}^{A}\right) d-d\right\|<\epsilon / 3$; hence

$$
\left\|\mathbb{X}_{1 \rho}\left(x_{i} \otimes d\right)-\mathbb{X}_{1 \rho}\left(x_{i} \otimes \rho\left(e_{\lambda^{\prime}}^{A}\right) d\right)\right\|<\epsilon / 3, \quad\left\|x_{i} \otimes \rho\left(e_{\lambda^{\prime}}^{A}\right) d-x_{i} \otimes d\right\|<\epsilon / 3
$$

for each $x_{i}$. Furthermore, by (4.5), there exists $i^{\prime}$ such that for $i \geq i^{\prime}$

$\left\|\mathbb{X}_{1 \rho}\left(x_{i} \otimes \rho\left(e_{\lambda^{\prime}}^{A}\right) d\right)-x_{i} \otimes \rho\left(e_{\lambda^{\prime}}^{A}\right) d\right\| \leq\|d\| \cdot \|\left(\mathbb{X}_{1 \rho}\left(x_{i} \otimes \rho\left(e_{\lambda^{\prime}}^{A}\right)\right)-x_{i} \otimes \rho\left(e_{\lambda^{\prime}}^{A}\right) \|<\epsilon / 3\right.$.

The last two estimates together give

$$
\begin{aligned}
& \left\|\mathbb{X}_{1 \rho}\left(x_{i} \otimes d\right)-\left(x_{i} \otimes d\right)\right\| \leq\left\|\mathbb{X}_{1 \rho}\left(x_{i} \otimes d\right)-\mathbb{X}_{1 \rho}\left(x_{i} \otimes \rho\left(e_{\lambda^{\prime}}^{A}\right) d\right)\right\|+ \\
& \quad\left\|\mathbb{X}_{1 \rho}\left(x_{i} \otimes \rho\left(e_{\lambda^{\prime}}^{A}\right) d\right)-x_{i} \otimes \rho\left(e_{\lambda^{\prime}}^{A}\right) d\right\|+\left\|x_{i} \otimes \rho\left(e_{\lambda^{\prime}}^{A}\right) d-x_{i} \otimes d\right\|<\epsilon \quad \text { for } i \geq i^{\prime} .
\end{aligned}
$$

Since $\epsilon>0$ is arbitrary, we get $\lim _{i}\left\|\mathbb{X}_{1 \rho}\left(x_{i} \otimes d\right)-\left(x_{i} \otimes d\right)\right\|=0$ for $d \in \mathcal{D}_{\mathrm{V}}$.

Similarly, for a net of almost invariant unit vectors $\left\{y_{j}\right\}$ in $\mathcal{H}_{2}$ for $\mathrm{Y}$, we have $\lim _{j}\left\|\mathbb{Y}_{1 \theta}\left(y_{j} \otimes d\right)-y_{j} \otimes d\right\|=0$ for $d \in \mathcal{D}_{\mathrm{V}}$.

Finally, we show that $\left\{x_{i} \otimes y_{j}\right\}$ is an almost invariant unit vector for $\mathbb{X}_{1 \rho} \mathbb{Y}_{2 \theta}$. We compute

$$
\begin{aligned}
& \left\|\mathbb{X}_{1 \rho} \mathbb{Y}_{2 \theta}\left(x_{i} \otimes y_{j} \otimes d\right)-x_{i} \otimes y_{j} \otimes d\right\| \\
& \leq\left\|\mathbb{X}_{1 \rho} \mathbb{Y}_{2 \theta}\left(x_{i} \otimes y_{j} \otimes d\right)-\mathbb{X}_{1 \rho}\left(x_{i} \otimes y_{j} \otimes d\right)\right\|+\left\|\mathbb{X}_{1 \rho}\left(x_{i} \otimes y_{j} \otimes d\right)-x_{i} \otimes y_{j} \otimes d\right\| .
\end{aligned}
$$

Therefore, $\lim _{i, j}\left\|\mathbb{X}_{1 \rho} \mathbb{Y}_{2 \theta}\left(x_{i} \otimes y_{j} \otimes d\right)-x_{i} \otimes y_{j} \otimes d\right\|=0$ for $d \in \mathcal{D}_{\mathrm{V}}$.

Corollary 4.6. Assume $\mathbb{G}$ and $\widehat{\mathbb{G}}$ are coamenable. Then the Drinfeld double $\mathfrak{D}(\mathbb{G})$ has the Haagerup property.

Proof. By [7, Proposition 5.2], both $\mathbb{G}$ and $\widehat{\mathbb{G}}$ have the Haagerup property; hence $\mathfrak{D}(\mathbb{G})$ has the Haagerup property.

Remark 4.7. The proof of Theorem 4.4 also works in more general framework of locally compact quantum groups constructed from manageable multiplicative unitaries [17. Furthermore, one can generalise the statement of Theorem 4.4 by replacing $\widehat{\mathbb{G}}$ by another locally compact quantum group $\mathbb{H}$ with the Haagerup property, and the Drinfeld of $\mathbb{G}$ by generalised Drinfeld double of $\mathbb{G}$ and $\mathbb{H}$ with respect to a given bicharacter (see [14]) between them. 
Proposition 4.8. The Drinfeld doubles of locally compact groups $G$ with the Haagerup property, coamenable compact quantum groups $\mathbb{G}$, extended quantum $\mathrm{SU}(1,1)$, quantum $a x+b$, quantum $a z+b$ and quantum $\mathrm{E}(2)$ groups have the Haagerup property, respectively. In particular, the Drinfeld doubles of $\mathbb{F}_{n}$ (free group with $n(\geq 2)$ generators) and the duals of quantum Lorentz groups have the Haagerup property.

Proof. Let $G$ be a locally compact group; hence $\mathrm{C}_{0}(G)$ is coamenable. By [7, Proposition 5.2], $\mathrm{C}_{\mathrm{r}}^{*}(G)$ (as a quantum group) has the Haagerup property. Furthermore, if $G$ has the Haagerup property if and only if $\mathrm{C}_{0}(G)$ has the Haagerup property. Consequently, by Theorem 4.4, the Drinfeld double of $G$, namely, $\mathrm{C}_{0}(G) \rtimes_{\text {conj }} G$ has the Haagerup property if and only if $G$ has the Haagerup property. In particular, the Drinfeld doubles of $\mathbb{F}_{n}$ (free group with $n(\geq 2)$ generators) have the Haagerup property.

It is shown in 6 ] that the extended quantum $\mathrm{SU}(1,1)$ groups have the Haagerup property. By [6, Theorem 8.3], their duals also have the Haagerup property. By Theorem 4.4 Drinfeld doubles of the extended $\mathrm{SU}_{q}(1,1)$ has the Haagerup property.

Let $\mathbb{G}$ be a compact quantum group. By, [3, Proposition 4.1], the discrete quantum group $\widehat{\mathbb{G}}$ is always coamenable. Additionally, if $\mathbb{G}$ is coamenable, by Corollary 4.6, $\mathfrak{D}(\mathbb{G})$ is amenable and has the Haagerup property. In particular, $\mathrm{SU}_{q}(2)$ is a compact quantum group and coamenable (see [5]). Hence, the Drinfeld double of $\mathrm{SU}_{q}(2)$, dual of the quantum Lorentz group in [13], has the Haagerup property.

Finally, [7, Example 5.4] shows that quantum $a x+b$, quantum $a z+b$ and quantum $\mathrm{E}(2)$ groups have the Haagerup property. Therefore, by Corollary 4.6 we conclude that their respective Drinfeld doubles have the Haagerup property.

\section{ACKNOWLEDGEMENTS}

This research was conducted at the Fields Institute during the thematic program on Abstract Harmonic Analysis, Banach and Operator Algebras while the author was financially supported by a Fields-Ontario postdoctoral fellowship. Apart from that, parts of the manuscript had been revised while the author had been additionally supported by NSERC and ERA at the University of Ottawa. The author gratefully thanks Professor Nico Spronk for his helpful discussions and comments.

\section{REFERENCES}

[1] Saad Baaj and Georges Skandalis, Unitaires multiplicatifs et dualité pour les produits croisés de $C^{*}$-algèbres, Ann. Sci. École Norm. Sup. (4) 26 (1993), no. 4, 425-488, available at http://www .numdam.org/item?id=ASENS_1993_4_26_4_425_0 MR 1235438

[2] Saad Baaj and Stefaan Vaes, Double crossed products of locally compact quantum groups, J. Inst. Math. Jussieu 4 (2005), no. 1, 135-173, doi: 10.1017/S1474748005000034 MR 2115071

[3] Erik Bédos, Gerard J. Murphy, and Lars Tuset, Amenability and coamenability of algebraic quantum groups, Int. J. Math. Math. Sci. 31 (2002), no. 10, 577-601, doi: 10.1155/S016117120210603X MR 1931751

[4] Erik Bédos and Lars Tuset, Amenability and co-amenability for locally compact quantum groups, Internat. J. Math. 14 (2003), no. 8, 865-884, doi: 10.1142/S0129167X03002046 MR 2013149

[5] Erik Bédos, Gerard. J. Murphy, and Lars Tuset, Co-amenability of compact quantum groups, J. Geom. Phys. 40 (2001), no. 2, 130-153, doi: 10.1016/S0393-0440(01)00024-9 MR 1862084

[6] Martijn Caspers, Weak amenability of locally compact quantum groups and approximation properties of extended quantum $\mathrm{SU}(1,1)$, Comm. Math. Phys. (2013), to appear. arXiv:1306.4558v2

[7] Matthew Daws, Pierre Fima, Adam G. Skalski, and Stuart A. White, The Haagerup property for locally compact quantum groups, J. Reine Angew. Math. (2014), to appear. arXiv:1303.3261v3

[8] V. G. Drinfel'd, Quantum groups, International Congress of Mathematicians (Berkeley, Calif., 1986), 1987, pp. 798-820. MR 934283 
[9] Erik Koelink and Johan Kustermans, A locally compact quantum group analogue of the normalizer of SU(1,1) in SL(2, C), Comm. Math. Phys. 233 (2003), no. 2, 231-296MR 1962042

[10] Johan Kustermans and Stefaan Vaes, Locally compact quantum groups, Ann. Sci. École Norm. Sup. (4) 33 (2000), no. 6, 837-934, doi: 10.1016/S0012-9593(00)01055-7 MR 1832993

[11] Tetsuya Masuda, Y. Nakagami, and Stanisław Lech Woronowicz, A $C^{*}$-algebraic framework for quantum groups, Internat. J. Math 14 (2003), no. 9, 903-1001, doi: 10.1142/S0129167X03002071 MR 2020804

[12] Ryszard Nest and Christian Voigt, Equivariant Poincaré duality for quantum group actions, J. Funct. Anal. 258 (2010), no. 5, 1466-1503, doi: 10.1016/j.jfa.2009.10.015 MR 2566309

[13] Piotr Podleś and Stanisław Lech Woronowicz, Quantum deformation of Lorentz group, Comm. Math. Phys. 130 (1990), no. 2, 381-431, available at http://projecteuclid.org/euclid.cmp/1104200517 MR 1059324

[14] Sutanu Roy, The Drinfeld double for $C^{*}$-algebraic quantum groups, J. Operator Theory (2015), accepted. arXiv:1404.5384v4

[15] Piotr M. Sołtan and Stanisław Lech Woronowicz, A remark on manageable multiplicative unitaries, Lett. Math. Phys. 57 (2001), no. 3, 239-252, doi: 10.1023/A:10122306298 1862455

[16] Stanisław Lech Woronowicz, Quantum E(2) group and its Pontryagin dual, Lett. Math. Phys. 23 (1991), no. 4, 251-263, doi: 10.1007/BF00398822 MR 1152695

[17] _ From multiplicative unitaries to quantum groups, Internat. J. Math. 7 (1996), no. 1, 127-149, doi: 10.1142/S0129167X96000086 MR 1369908

[18] _ Compact quantum groups, Symétries quantiques (Les Houches, 1995), North-Holland, Amsterdam, 1998, pp. 845-884. MR 1616348

[19] _ From multiplicative unitaries to quantum groups, Internat. J. Math. 7 (1996), no. 1, 127-149, doi: 10.1142/S0129167X96000086 MR 1369908

[20] _ Quantum ' $a z+b^{\prime}$ ' group on complex plane, Internat. J. Math. 12 (2001), no. 4, 461-503, doi: 10.1142/S0129167X01000836 MR 1841400

[21] Stanisław Lech Woronowicz and Stanisław Zakrzewski, Quantum 'ax $+b$ ' group, Rev. Math. Phys. 14 (2002), no. 7-8, 797-828, doi: 10.1142/S0129055X02001405 MR 1932667

E-mail address: sr26@u0ttawa.ca

Department of Mathematics and Statistics, 585 King Edward, K1N 6N5 Ottawa, CANADA 